\title{
Primordial Fluctuations from Inflation: a Consistent Histories Approach
}

\author{
David Polarski
}

\author{
Lab. de Mathématique et Physique Théorique, EP93 CNRS \\ Université de Tours, Parc de Grandmont, F-37200 Tours (France) \\ Département d'Astrophysique Relativiste et de Cosmologie, \\ Observatoire de Paris-Meudon, 92195 Meudon cedex (France)
}

August 12, 2018

\begin{abstract}
We show how the quantum-to-classical transition of the cosmological fluctuations produced during an inflationary stage can be described using the consistent histories approach. We identify the corresponding histories in the limit of infinite squeezing. To take the decaying mode into account, we propose an extension to coarse-grained histories.

PACS Numbers: 04.62.+v, 98.80.Cq
\end{abstract}




\section{Introduction}

In the inflationary paradigm [1], all inhomogeneities in the universe originated from primordial quantum fluctuations of some scalar field(s), the so-called inflaton(s). It is then possible to calculate these fluctuations [2], to track their evolution untill today and to make predictions, for each given model, concerning the formation of large-scale structures in the universe and the anisotropy of the Cosmological Microwave Background. The latter will be measured with high accuracy till small angular scales (see for example [3]) A comprehensive understanding of the quantum-to-classical transition of these fluctuations is required is order to explain the obviously classical nature of the inhomogeneities, both in matter and radiation, observed on cosmological scales. It was already shown that the fluctuations undergo a quantum-to-clasical transition as a result of their peculiar dynamics due to the accelerated expansion of the universe during the inflationary stage [4]. This dynamics leads to a very highly squeezed state [5, 6, 7], where the squeezing parameter $\left|r_{k}\right|$ satisfies $\left|r_{k}\right| \gg 1$. This is expressed in the Heisenberg representation by a vanishingly small decaying mode, nowadays on cosmological scales, compared to the growing mode. This is why inflationary fluctuations that are in principle observable today, after they reenter the Hubble radius, have a stochastic amplitude but a fixed phase. Note that random amplitude and fixed phase does not point necessarily to a quantum origin, but rather to a primordial origin [8, [] of the fluctuations. The inflationary paradigm explains elegantly this primordial origin. This has observational consequences, for instance the existence of secondary acoustic (Sakharov) peaks in the small-angle Cosmological Microwave Background anisotropy. The amplitudes have, in most models where the fluctuations arise from vacuum initial states, a Gaussian distribution. However the above results can be extended to a non-Gaussian distribution when fluctuations are produced in non vacuum initial sates [9]. In other words, this quantum-to-classical transition is essentially independent of the initial state. The sensitivity of this process to the environment was

also considered [10] and the result was found that for a wide class of interactions this transition still holds, independently of the fluctuations initial state at the inflation- 
ary stage, the amplitude basis defining the pointer basis. This quantum-to-classical transition that takes place solely as a result of the dynamics is very intriguing. The remaining coherence of the initial quantum state can be described to very high accuracy with classical stochastic terms. In the cosmological context one is certainly willing to accept the probabilistic description of the fluctuations due to an indeterminacy in the initial conditions. This fact really becomes non-trivial when the fluctuations are quantized. In that case, a deterministic evolution of the wave function leads effectively to a classical evolution with stochastic initial conditions. The non-relativistic free quantum particle at very late times provides yet another surprising example where an analogous transition is taking place [11]. We present here a way to describe this transition using the consistent histories approach 12 (see also 13 for a review). We feel that the consistent histories approach is conceptually very appropriate for the description of the quantum-to-classical transition taking place for the primordial fluctuations of inflationary origin. Furthermore, the Heisenberg picture which is used for the description of the (second quantized) fluctuations, enters in a natural way in this picture too. We first briefly review this approach and we show how, for the inflationary fluctuations, histories can be defined.

\section{Decoherent histories}

In this formalism, the evolution of a quantum system is given by specifying a set of alternative histories. These are defined by a set of Heisenberg projection operators $P_{\alpha_{i}}^{i}\left(t_{i}\right)$, at a sequence of times $t_{i}$ with $t_{1}<t_{2}<\ldots<t_{n}$. A single history corresponds to a particular choice $\alpha_{i}$ for each time $t_{i}$, i.e. to a particular set of indices $\{\alpha\} \equiv$ $\left\{\alpha_{1}, \alpha_{2}, \ldots, \alpha_{n}\right\}$. The Heisenberg projection operators $P_{\alpha_{i}}^{i}\left(t_{i}\right)$ satisfy the following conditions

$$
P_{\alpha_{i}}^{i}\left(t_{i}\right) P_{\alpha_{i}^{\prime}}^{i}\left(t_{i}\right)=P_{\alpha_{i}}^{i}\left(t_{i}\right) \delta_{\alpha_{i} \alpha_{i}^{\prime}}
$$

and

$$
\sum_{\alpha_{i}} P_{\alpha_{i}}^{i}=I d
$$


Condition (1), which says that the projection operators are orthogonal, means that the histories $\{\alpha\}$ are exclusive, while condition (2), which says that the projection operators $P_{\alpha_{i}}^{i}\left(t_{i}\right)$ form a complete set at each time $t_{i}$, means that the set of alternative histories is exhaustive. We can now define a history operator $C_{\alpha}$

$$
C_{\alpha} \equiv P_{\alpha_{n}}^{n} P_{\alpha_{n-1}}^{n-1} \ldots P_{\alpha_{2}}^{2} P_{\alpha_{1}}^{1}
$$

which gives the branch state vector

$$
C_{\alpha}|\Psi\rangle
$$

when applied to the initial state vector $\Psi$, where we assume that we start with a pure state (the case of relevance for inflation). Sets of alternative histories with vanishing interference are said to decohere. In that case, it is possible to attach consistently a probability $p(\alpha)$ to each individual history $\{\alpha\}$

$$
p(\alpha)=\left\langle\Psi\left|C_{\alpha}^{\dagger} C_{\alpha}\right| \Psi\right\rangle
$$

Interference is mesured by the decoherence functional $D\left(\alpha \alpha^{\prime}\right)$

$$
D\left(\alpha \alpha^{\prime}\right)=\left\langle\Psi\left|C_{\alpha}^{\dagger} C_{\alpha^{\prime}}\right| \Psi\right\rangle
$$

Hence we have in the case of decoherent histories

$$
D\left(\alpha \alpha^{\prime}\right)=p(\alpha) \delta_{\alpha \alpha^{\prime}}
$$

These definitions are straightforewardly generalized when one starts with a mixed state and the corresponding density matrix $\rho$. In that case we have

$$
D\left(\alpha \alpha^{\prime}\right)=\operatorname{Tr}\left(C_{\alpha} \rho C_{\alpha^{\prime}}^{\dagger}\right)=p(\alpha) \delta_{\alpha \alpha^{\prime}}
$$

\section{Quantum fluctuations from inflation}

Let us consider now the primordial quantum fluctuations produced during the inflationary stage. Regarding the quantum-to-classical transition, the tensorial fluctuations (or gravitational waves) can serve as the paradigm for both types of fluctuations, 
scalar and tensorial, of inflationary origin. The physics of these quantum fluctuations was already studied in depth, we give now the essential results using the notations of 4 . The quantities $y_{\mathbf{k}}$, resp. $p_{\mathbf{k}}$, are the Fourier tansforms of the amplitude $y$, resp. the conjugate momentum $p$, all these quantities being time-dependent. The conformal time $\eta \equiv \int^{t} \frac{d t^{\prime}}{a\left(t^{\prime}\right)}$ is used.

The dynamics of the system is particularly transparent in the Heisenberg representation. We have, using the amplitude field modes $f_{k}(\eta)$ with $\Re f_{k} \equiv f_{k 1}$ and $\Im f_{k} \equiv f_{k 2}, f_{k}\left(\eta_{0}\right)=1 / \sqrt{2 k}$, (we adopt a similar notation for all quantities)

$$
\begin{aligned}
y(\mathbf{k}, \eta) & \equiv f_{k}(\eta) a\left(\mathbf{k}, \eta_{0}\right)+f_{k}^{*}(\eta) a^{\dagger}\left(-\mathbf{k}, \eta_{0}\right) \\
& =f_{k 1}(\eta) e_{y}(\mathbf{k})-f_{k 2}(\eta) e_{p}(\mathbf{k})
\end{aligned}
$$

and the momentum field modes $g_{k}(\eta), g_{k}\left(\eta_{0}\right)=\sqrt{\frac{k}{2}}$,

$$
\begin{aligned}
p(\mathbf{k}, \eta) & \equiv-i\left[g_{k}(\eta) a\left(\mathbf{k}, \eta_{0}\right)-g_{k}^{*}(\eta) a^{\dagger}\left(-\mathbf{k}, \eta_{0}\right)\right] \\
& =g_{k 1}(\eta) e_{p}(\mathbf{k})+g_{k 2}(\eta) e_{y}(\mathbf{k})
\end{aligned}
$$

The time independent operators $e_{p}(\mathbf{k}) \equiv \sqrt{\frac{2}{k}} p\left(\mathbf{k}, \eta_{0}\right), \operatorname{resp} . \quad e_{y}(\mathbf{k}) \equiv \sqrt{2 k} y\left(\mathbf{k}, \eta_{0}\right)$, satisfy

$$
\left\langle e_{y}(\mathbf{k}) e_{y}^{\dagger}\left(\mathbf{k}^{\prime}\right)\right\rangle=\left\langle e_{p}(\mathbf{k}) e_{p}^{\dagger}\left(\mathbf{k}^{\prime}\right)\right\rangle=\delta^{(3)}\left(\mathbf{k}-\mathbf{k}^{\prime}\right) \quad e_{y, p}^{\dagger}(\mathbf{k})=e_{y, p}(-\mathbf{k})
$$

They obey the commutation relations

$$
\left[e_{i}(\mathbf{k}), e_{j}^{\dagger}\left(\mathbf{k}^{\prime}\right)\right]=2 i \delta_{i j} \delta^{(3)}\left(\mathbf{k}-\mathbf{k}^{\prime}\right), \quad i, j=y, p
$$

When the initial state is the vacuum state, all (non vanishing) correlation functions are derived from (11). The field modes can be parametrized by three parameters, the squeezing parameter $r_{k}$, the squeezing angle $\phi_{k}$ and the rotation angle $\theta_{k}$.

If $\lambda$ is the physical wavelength of the perturbation, $R_{H} \equiv \frac{a}{\dot{a}}$ (in units for which $c=1$ ) the Hubble radius and $a(t)$ the scale factor of the FRW metric then, time evolution in the regime $\lambda \gg R_{H}$ leads to an extreme squeezing which persists when the perturbation reenters the Hubble radius, i.e. for $\lambda<R_{H}$. As a result, it is possible to take $f_{k 2} \rightarrow 0$ and $g_{k 1} \rightarrow 0$ (see e.g. [4]). The quantum coherence is then expressible 
in classical stochastic terms: for a given "realization" $y_{\mathbf{k}}$ of the fluctuation field, we have for its canonical momentum $p_{\mathbf{k}} \simeq \frac{g_{k 2}}{f_{k 1}} y_{\mathbf{k}} \equiv p_{\mathbf{k}, c l}$, the classical momentum for large squeezing, i.e., for $\left|r_{k}\right| \rightarrow \infty$. This almost perfect classical correlation is nicely exhibited with the help of the Wigner function - a well-known example of (quasi) probability density in phase-space $4,14,15]$.

We consider now the operators $y_{\mathbf{k}}$ separately for each $\mathbf{k}$ but, for brevity of notation, we will drop in the following the subscript $\mathbf{k}$ (or $\mathrm{k}$ ). In the limit of a perfect classical correlation, the system is described by a set of decoherent histories, to each single history a probability can be assigned, these probabilities add to one. The projection operators defining the histories in that case are given by

$$
P_{\alpha_{i}}^{i}\left(t_{i}\right)=\left|y_{i}\right\rangle\left\langle y_{i}\right|
$$

where $y_{i}$ stands for a particular value of the operator $y(t)$ at time $t_{i}$. We can now consistently assign a probability (density) to each individual trajectory $\left(y\left(t_{n}\right) y\left(t_{n-1}\right) \ldots y\left(t_{1}\right)\right)$ in amplitude space, where all the $y\left(t_{i}\right)$ lie on the classical trajectory (for $\left|r_{k}\right| \rightarrow \infty$ ) that goes through $y\left(t_{1}\right)$ at time $t_{1}$. Indeed, probability is conserved along classical trajectories passing through $y_{i}$ (at some late time $t_{i}$ ), with

$$
p(\alpha) \equiv\left|\Psi\left(y_{0}, \eta_{0}\right)\right|^{2} \quad y_{i}=f_{k 1}\left(\eta_{i}\right) y_{0} \equiv y_{i, c l} \quad \forall i
$$

In the limit where the decaying mode is negligible, i.e. $f_{k 2} \rightarrow 0$, (14) holds for all realizations $y_{i}$ at time $t_{i}$ of the amplitude. This is the highly non-trivial property of our (isolated) pure state allowing for its description in classical terms. In particular, we can consistently define the joint probability

$$
W\left(y_{n}, t_{n} ; y_{n-1}, t_{n-1} ; \ldots ; y_{2}, t_{2} ; y_{1}, t_{1}\right) d y_{n} \ldots d y_{1}
$$

to find the amplitude $y$ between $y_{i}$ and $y_{i}+d y_{i}$ at each late time $t_{i}$. Of course this joint probability is Markovian and satisfies

$$
\begin{array}{r}
W\left(y_{n}, t_{n} ; y_{n-1}, t_{n-1} ; \ldots ; y_{2}, t_{2} ; y_{1}, t_{1}\right)=p\left(y_{n}, t_{n} ; y_{n-1}, t_{n-1}\right) \times \\
p\left(y_{n-1}, t_{n-1} ; y_{n-2}, t_{n-2}\right) \ldots p\left(y_{2}, t_{2} ; y_{1}, t_{1}\right) \mathcal{P}\left(y_{1}, t_{1}\right)
\end{array}
$$


where $p\left(y_{i}, t_{i} ; y_{i-1}, t_{i-1}\right)$ is the conditional probability density to have $y\left(t_{i}\right)=y_{i}$ provided we had $y\left(t_{i-1}\right)=y_{i-1}$. Further, $\mathcal{P}\left(y_{1}, t_{1}\right)$ is the probability for $y\left(t_{1}\right)=y_{1}$ and it satisfies

$$
\mathcal{P}\left(y_{1}, t_{1}\right)=\left|\Psi\left(y_{0}, \eta_{0}\right)\right|^{2}=p(\alpha) \quad y_{1}=f_{k 1}\left(\eta_{1}\right) y_{0}
$$

Actually, we have very generally

$$
p\left(y_{i}, t_{i} ; y_{j}, t_{j}\right)=\delta\left(y\left(t_{i}\right)-\frac{f_{1}\left(t_{i}\right)}{f_{1}\left(t_{j}\right)} y_{j}\right) \quad 1 \leq j \leq n
$$

Equation (19) is valid in the limit that the decaying mode is negligible. The consistent histories picture exhibits nicely the classical stochastic nature of the fluctuations: in the limit $\left|r_{k}\right| \rightarrow \infty$ one should not expect to find the system on one single classical trajectory. Rather, it can be found on any classical trajectory with a certain probability. Each of these classical trajectories constitutes one of the possible alternative histories to which a probability can be assigned. Usually only probability amplitudes can be assigned to trajectories due to quantum interference [16]. While the precise physical meaning of the individual trajectories is a subject of (endless) debate, the crucial point here is the ability to consistently assign a probability to each history ("classical trajectory").

For large enough, though nevertheless finite squeezing parameter $\left|r_{k}\right|$, which is the case relevant for fluctuations of inflationary origin, one may wish to take the decaying mode into account. In that case we suggest a more realistic description of our system in terms of coarse-grained histories corresponding to coarse-grained trajectories in amplitude space. Instead of assigning a certain probability to the history $\left(y\left(t_{n}\right) y\left(t_{n-1}\right) \ldots y\left(t_{1}\right)\right)$, we now assign a probability to the history $\left(\Delta y\left(t_{n}\right) \Delta y\left(t_{n-1}\right) \ldots \Delta y\left(t_{1}\right)\right)$ where the projection operator $P_{\alpha_{i}}^{i}\left(t_{i}\right)$ is now given by

$$
P_{\alpha_{i}}^{i}\left(t_{i}\right)=\left|\Delta y_{i}\right\rangle\left\langle\Delta y_{i}\right|
$$

where $\left|\Delta y_{i}\right\rangle$ corresponds to a state for which the amplitude $y(t)$ at time $t_{i}$ is localized within the interval $\Delta y_{i}$. The corresponding decoherence functional $D_{\Delta}\left(\alpha \alpha^{\prime}\right)$ can then be written in the following suggestive way

$$
D_{\Delta}\left(\alpha \alpha^{\prime}\right)=\int_{\alpha} \mathcal{D} y \int_{\alpha^{\prime}} \mathcal{D} y^{\prime} D\left(y(t), y^{\prime}(t)\right)
$$


where $D\left(y(t), y\left(t^{\prime}\right)\right)$ corresponds to the histories constructed with the projection operators $|y(t)\rangle\langle y(t)|$ and $\{\alpha\} \equiv\left(\Delta y_{n}, \ldots, \Delta y_{1}\right)$. Decoherence is achieved when

$$
D_{\Delta}\left(\alpha \alpha^{\prime}\right) \approx 0
$$

We can think of a particular history as a series of slits trough which our system has to pass at the successive times $t_{i}$, the condition (22) then expresses the almost complete absence of diffraction. This almost complete absence of diffraction was shown with a slit (thought) experiment in [15]. Note that the integrals appearing in (21) are really functional integrals, integrals over all the possible paths 17 that can belong to a given coarse-grained history $\{\alpha\}$, not just classical paths. In inflation we will have that $\Delta y_{i}$ is tremendously small, far beyond our observational capabilities. This is where the smallness of the decaying mode enters.

To summarize, we have shown how the quantum-to-classical transition of quantum fluctuations of inflationary origin can be nicely described in the consistent histories picture. We have identified the histories and the corresponding projection operators in the limit $\left|r_{k}\right| \rightarrow \infty$ and we have extended our description, using coarse grained histories, to the more realistic case where the decaying mode, though tremendously tiny compared to the growing mode, is nevertheless present. In the case of fluctuations arising from inflation, the decaying mode is definitely negligible compared to the growing mode so that the limit $\left|r_{k}\right| \rightarrow \infty$ safely applies for most purposes. However, depending on the level of precision with which one is willing, or has, to describe the fluctuations one may wish to take the decaying mode into account. We propose to use coarse-grained histories for this purpose. In a sense, it amounts to describe the evolution of our system in terms of classical trajectories with a very tiny quantum noise which is due to that piece of the amplitude ("position") operator connected to the decaying mode. It is also possible to consider decoherence in the systemenvironment couple in the consistent histories picture [18] and we leave these points for further investigation. The consistent histories picture is known to be very fruitfull in many problems related to decoherence in particular, and to the interpretation of quantum mechanics in general. It is therefore gratifying that this approach can be 
used in the context of inflation as well. In particular all the interpretational questions arising in this picture can be considered for the particular, but all-important, case of fluctuations of inflationary origin.

\section{References}

[1] A. Linde, Rep. Prog. Phys. 47 (1984) 925; Particle physics and inflationary cosmology (Harwood, New York, 1990); E. Kolb, M. Turner, The Early Universe (Addison-Wesley, Redwood City, 1990).

[2] S.W. Hawking, Phys. Lett. B 115 (1982) 295; A.A. Starobinsky, Phys. Lett. B 117 (1982) 175; A.H. Guth and S-Y. Pi, Phys. Rev. Lett. 49 (1982) 1110.

[3] http://astro.estec.esa.nl/SA-general/Projects/Planck/ http://map.gsfc.nasa.gov/

[4] D. Polarski and A.A. Starobinsky, Class. Quantum Grav. 13 (1996) 377.

[5] L.P. Grishchuk and Y.V. Sidorov, Phys. Rev. D 42 (1990) 3413.

[6] A. Albrecht, P. Ferreira, M. Joyce, and T. Prokopec, Phys. Rev. D 50 (1994) 4807.

[7] A. Albrecht, Report hep-th/9402062.

[8] D. Polarski and A.A. Starobinsky, Phys. Lett. B 356 (1995) 196.

[9] J. Lesgourgues, D. Polarski, and A.A. Starobinsky, Nucl. Phys. B 497 (1997) 479.

[10] C. Kiefer, D. Polarski, and A.A. Starobinsky, Int. Journ. Mod. Phys. D 7 (1998) 455.

[11] Kiefer C and Polarski D Report gr-qc/9805014, Ann. Physik 7 (1998) 137. 
[12] R. Griffiths, J. Stat. Phys. 36 (1984) 219.

R. Griffiths, Phys. Rev. A 54 (1996) 2759.

R. Omnès, J. Stat. Phys. 53 (1988) 893.

R. Omnès, The interpretation of Quantum Mechanics (Princeton University Press, Princeton 1994).

M. Gell-Mann and J. Hartle, Complexity, Entropy and the Physics of Information, SFI Studies in the Sciences of Complexity, Vol.VIII, (Edited by W. Zurek) (Addison Wesley, Reading 1990), p.425.

[13] Giulini D, Joos E, Kiefer C, Kupsch J, Stamatescu I O and Zeh H D, Decoherence and the Appearance of a Classical World in Quantum Theory (Springer 1996).

[14] J. Lesgourgues, D. Polarski, and A.A. Starobinsky, Class. Quantum Grav. 14 (1997) 881.

[15] C. Kiefer, J. Lesgourgues, D. Polarski, and A.A. Starobinsky, Class. Quantum Grav. 15 (1998) L67.

[16] Feynman R P and Hibbs A R, Quantum Mechanics and Path Integrals (McGrawHill 1965).

[17] J. Hartle Phys. Rev. D 10 (1991) 3173.

[18] J. Finkelstein Phys. Rev. D 47 (1993) 5430. 